This is a self-archived version of an original article. This version may differ from the original in pagination and typographic details.

Author(s): Huang, Jun; Liao, Yong; Xing, Cong-Cong; Chang, Zheng

Title: Multi-hop D2D Communications with Network Coding : From A Performance Perspective

Year: 2019

Version: Accepted version (Final draft)

Copyright: @ 2019 IEEE.

Rights: In Copyright

Rights url: http://rightsstatements.org/page/InC/1.0/?language=en

Please cite the original version:

Huang, J., Liao, Y., Xing, C.-C., \& Chang, Z. (2019). Multi-hop D2D Communications with Network Coding : From A Performance Perspective. IEEE Transactions on Vehicular Technology, 68(3), 2270-2282. https://doi.org/10.1109/TVT.2019.2890952 


\title{
Multi-hop D2D Communications with Network Coding: From a Performance Perspective
}

\author{
Jun Huang, Senior Member, IEEE Yong Liao, Cong-cong Xing, and Zheng Chang, Senior Member, IEEE
}

\begin{abstract}
Multi-hop D2D communications play an important role in expanding D2D coverage. In this paper, we study a relaybased and network-coding-assisted (in particular, XOR coding) multi-hop D2D communication system. In the system, toward jointly considering the impact of interference and network traffic conditions on the quality of D2D communications, various channel fading models and traffic models are investigated, and the packet loss probability of D2D links is meticulously computed using these models. With packet loss probability of D2D links, the general closed-form expressions of end-to-end packet loss probability (E2EPLP) of the system with the presence (or absence) of XOR coding are subsequently derived. Our experiments reveal that both the number of relays and the mechanism of XOR coding can affect the system performance. Specifically, the increase in the number of relays will lower the overall system performance (e.g., an increase in the E2EPLP and end-to-end completion time, and a decrease of the end-to-end rate may follow as a result). On the other hand, although the presence of XOR coding unfortunately raises the system E2EPLP, it can effectively improve the end-toend completion time and end-to-end rate. It is our belief that the analytical approach proposed in this paper and the results found in our work can be useful to peer studies in the context of applying network coding in multi-hop D2D networks.
\end{abstract}

Index Terms-multi-hop D2D communication, network coding, traffic model, performance evaluation.

\section{INTRODUCTION}

The paradigm of device-to-device (D2D) communications underlaying cellular networks has been proposed to address the dramatically increasing Internet access demands from mobile users. This paradigm is widely considered as an effective way to improve transmission rate, to reduce transmission latency and power consumption, and to enhance spectrum efficiency [1]. Unfortunately, the direct single-hop D2D communication mechanism only works well within a region of a short radius, and has trouble in delivering quality transmissions over a large area. Toward remedying this, the idea of multihop D2D communications has been proposed and investigated to expand the D2D coverage [2].

Relay nodes are used in multi-hop D2D communications to forward data packets. On the one hand, this mechanism can expand the signal communication range, which makes D2D communications be able to fit complex and diversified

This research is supported by NSFC under grant number 61671093 .

J. Huang and Y. Liao are with the Chongqing University of Posts and Telecommunications, Chongqing, 400065 China, e-mail: xiaoniuadmin@gmail.com.

C. Xing is with the Department of Mathematics and Computer Science, Nicholls State University, Thibodaux, LA 70310, USA, e-mail: congcong.xing@nicholls.edu.

Z. Chang is with the Faculty of Information Technology, University of Jyvaskyla, FI-40014 Jyvaskyla, Finland. e-mail: zheng.chang@jyu.fi. environments. On the other hand, it can also shorten the distance of D2D links per hop, which may eventually reduce the transmit power and energy consumption. Incidentally, the relay selection strategy is of paramount significance in multihop D2D communications. In the area of cooperative communications, relay techniques have been studied comprehensively. For example, [3] studied a social relationship to effectively select relay users, [4] proposed a social-aware energy-efficient relay selection mechanism that considers the hidden social ties among mobile users. Also, a relay selection policy based on the buffering ability of relay nodes was presented in [5], and the random distribution of relay nodes by treating the relay deployment as a Poisson point process was investigated in [6]. In this paper, we assume that relay users are able to and willing to forward data packets, and devise a primitive relay selection strategy that is based on the location information of users in the multi-hop D2D communication scenario. In this strategy, the base station selects the relay users in such a way that the sum of the distances from the selected relay to the two D2D ends is minimal.

It is known that network coding is capable of improving network throughput performance by allowing relay nodes to compute and then forward the received packets [7], [8], [9]. Generally speaking, the techniques of network coding can be divided into the following major categories [10]: physical layer network coding (PNC), analog network coding (ANC), instant decodable network coding (IDNC), and random linear network coding (RLNC). Each network coding method has its own pros and cons that fit best with a particular scenario.

Many efforts have been made to analyze the performance of network coding or relay assisted D2D communications. [11] analyzed the average energy efficiency and spectral efficiency by assuming that the signal fading is Rayleigh fading. [12] and [13] studied the problem of resource allocation for network coding aided D2D communications. The completion time required for recovering all missing packets at devices using IDNC was investigated in [14] and [15]. The authors of [16] developed a radio resource management (RRM) mechanism and investigated how caching capabilities at relay nodes would impact the system performance. While [17] evaluated the performance of the network coding aided D2D communications in terms of throughput, energy efficiency, and battery consumption, [18] devised a game-theoretic model for the compensation power acquisition of D2D transmitters. [19] considered a new class of codes (fountain codes) to keep reliable transmissions of data in multi-hop erasure channels. [20] proposed a reliable multicast protocol which is built upon opportunistic routing and random linear network coding. Furthermore, the 
closed-form expressions of outage probabilities were obtained for relay-assisted OFDM D2D systems in [21]. The network throughput and end-to-end packet loss probability (E2EPLP) were evaluated for multi-hop D2D communications in [22]. Differing from previous studies, we procure the generic closedform expressions of E2EPLP with the presence or absence of the network coding, respectively, on the basis of the packet loss probability (PLP) of D2D links. Also, considering that some existing studies [22], [23] only investigate the special case of one relay node (two-hop) D2D communications, we extend the prior studies by considering the general case of an arbitrary number of hops in our work.

Note that network transmissions typically experience intermittent interference or noise causing unrecoverable errors in packets that are then discarded at the receivers, we need to choose a suitable channel fading model to analyze the PLP of D2D links. In most of the prior studies, the Rayleigh fading model, which ignores D2D communications in the line-ofsight $(\mathrm{LoS})$, is adopted. This, in fact, is problematic in the sense that close-range communications (e.g., D2D communications) often lead to the existence of dominant LoS signal components in the received desired signals [24], [25], [26]. In addition, as packet losses occur frequently due to network traffic congestions that cause packet buffer overflows in relay nodes, there is a need to select an appropriate traffic model to analyze the queue capacity and the service capability of relay nodes in multi-hop D2D communications. This has also been extensively explored in the literature. For example, the packet arrival rate, when the packet arrival in D2D communications is treated as a Poisson arrival process, is studied in [27]; a dynamic D2D traffic model and the ensuing queue delay of D2D communications are investigated in [28]; and a dynamic data arrival process with non-saturated buffers is studied in [29]. As such, we in our work investigate the system performance in different channel fading models (Rician-Rayleigh and Rayleigh-Rayleigh) and in different traffic models (Pareto, fractional Brownian motion, and Poisson).

Our contributions in this paper are summarized as follows.

- A general multi-hop D2D communication model underlaying the cellular network is introduced, which is followed by the formulation of the related channel fading models and traffic models. We propose a primitive relay selection strategy, in which the base station selects the relay nodes using their polar coordinates information and by the criterion of the "shortest path" (see Section II-D). Moreover, a coding scheme where packets are encoded by network coding at the relay nodes is investigated for the data transmission over multi-hop D2D links.

- By jointly considering the impact of interference and network traffic conditions on the quality of D2D communications, the packet loss probability of D2D links is carefully constructed from two perspectives - the outage probability and packet failure probability, which are subsequently computed by using the Rician-Rayleigh fading model and by using different traffic models.

- The closed-form expressions of the system E2EPLP with the presence or absence of the network coding are obtained on the basis of the packet loss probability of D2D
TABLE I: LIST OF IMPORTANT NOTATIONS

\begin{tabular}{|c|c|}
\hline Notation & Meaning \\
\hline$M$ & The number of relay nodes \\
\hline$N_{1}, N_{2}$ & $\begin{array}{l}\text { The number of source packets correctly received by nodes } \\
M+1 \text { and } 0 \text {, respectively. }\end{array}$ \\
\hline$K$ & The Rician factor \\
\hline$(i, j)$ & The transmission link from node $i$ to node $j$ \\
\hline$\lambda_{j}$ & The average packet arrival rate at node $j$ \\
\hline$\mu_{j}$ & The average service rate at node $j$ \\
\hline$\Delta T$ & The fixed duration of each time slot \\
\hline$A_{j}(\Delta T)$ & $\begin{array}{l}\text { The cumulative arrival amount at node } j \text { during each time } \\
\text { slot }\end{array}$ \\
\hline$Z_{j}$ & The maximum queue capacity of node $j$ in terms of packets \\
\hline$Q_{j}(t)$ & The queue length of node $j$ at the beginning of $t$-th time slot \\
\hline$\gamma_{0}$ & The SINR threshold at any node \\
\hline$\sigma^{2}$ & Additive white Gaussian noise \\
\hline$\theta$ & A variable in $\mathrm{dB}, \theta=\frac{P_{T_{i}} \cdot d_{i, j}{ }^{-\alpha} \cdot \Omega}{P_{T_{M+2}} \cdot d_{M+2, j}-\alpha \cdot \omega+\sigma^{2}}$ \\
\hline$T_{s}^{c}$ & The total time slots with network coding \\
\hline$T_{E 2 E}^{c}$ & The end-to-end completion time with network coding \\
\hline$R_{E 2 E}^{c}$ & The average end-to-end transmission rate with network coding \\
\hline $\operatorname{Pr}_{i, j}^{l}$ & The packet loss probability of link $(i, j)$ \\
\hline $\operatorname{Pr}_{i, j}^{s}$ & $\begin{array}{l}\text { The packet success probability of link }(i, j), \operatorname{Pr}_{i, j}^{s}=1- \\
\operatorname{Pr}_{i, j}^{l}\end{array}$ \\
\hline $\operatorname{Pr}_{i, j}^{o}$ & The outage probability of link $(i, j)$ \\
\hline$\hat{\operatorname{Pr}}_{i, j}$ & The packet delivery probability over link $(i, j)$ \\
\hline $\begin{array}{l}\overline{P r}_{i, j} \\
\operatorname{Pr}_{i}^{c}\end{array}$ & $\begin{array}{l}\text { The packet failure probability, } \overline{\operatorname{Pr}}_{i, j}=1-\hat{\operatorname{P}} r_{i, j} \\
\text { The probability that node } i \text { can encode packets }\end{array}$ \\
\hline $\operatorname{Pr}_{i, j}^{l, c}$ & The packet loss probability of link $(i, j)$ with network coding \\
\hline $\operatorname{Pr}_{E 2 E}^{l, c}$ & The end-to-end packet loss probability with network coding \\
\hline $\operatorname{Pr}_{E 2 E}^{l}$ & $\begin{array}{l}\text { The end-to-end packet loss probability without network cod- } \\
\text { ing }\end{array}$ \\
\hline
\end{tabular}

links. We find that both the number of relays and network coding have impacts on the system E2EPLP, end-to-end completion time, and average end-to-end transmission rate, which are critical performance indicators of the system.

The rest of this paper is organized as follows. Section II introduces the system model of multi-hop D2D communications underlaying the cellular network, and the associated channel models and traffic models. The D2D transmission with network coding and its packet loss probability are investigated in Section III. The general closed-form expressions of E2EPLP with or without network coding and the related performance indicators are given in Section IV. The simulation results validating these performance indicators are provided in Section V. Section VI concludes the paper. Also, notations used in the paper are summarized in Table I.

\section{System Model}

\section{A. Network model}

As shown in Fig. 1, we consider a general scenario of multihop D2D communications underlaying cellular networks. In the cell shown in Fig. 1, there are some randomly distributed cellular user equipment $(C U E)$ and a pair of D2D user equipment $\left(D U E_{1}\right.$ and $\left.D U E_{2}\right)$. These two D2D users need to exchange some multimedia contents like pictures, live videos, or interactive games. It is assumed that the distance between $D U E_{1}$ and $D U E_{2}$ is too long for $D U E_{1}$ and $D U E_{2}$ to directly communicate with each other using a one-hop D2D 


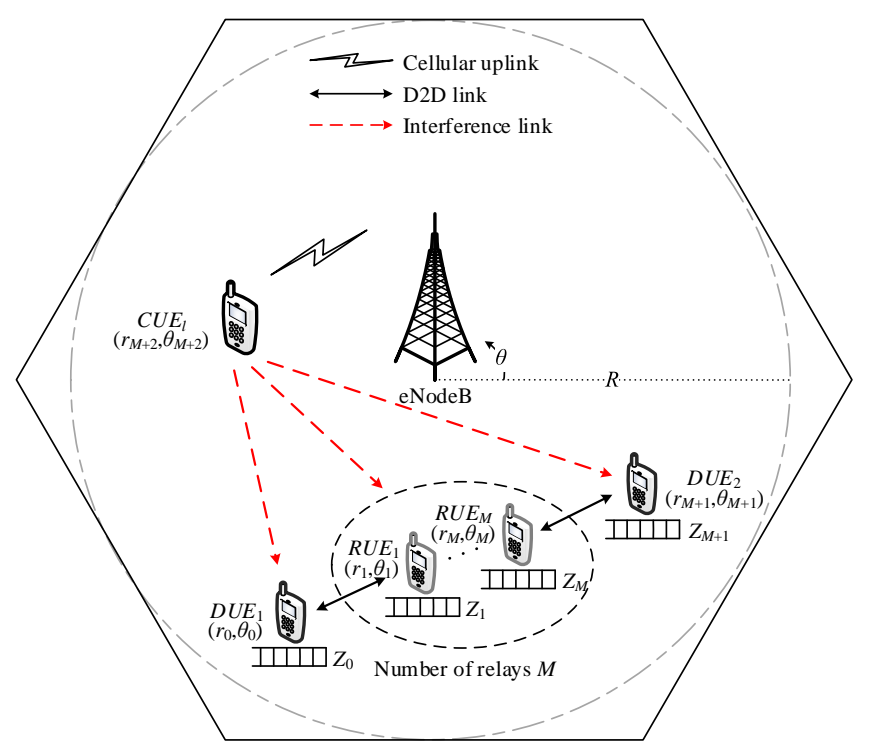

Fig. 1: Multi-hop D2D communications underlaying cellular networks.

link. Therefore, some relay user equipment $(R U E)$ need to be added to establish multi-hop D2D links between $D U E_{1}$ and $D U E_{2}$. We assume that the number of $R U E$ is $M$ and all of these relay users can be treated essentially as D2D users. The scheduling and resource allocation for all D2D user equipment $\left(D U E_{1}, D U E_{2}\right.$ and $R U E$ ) can be done by the base station eNodeB. Also, it is assumed that all $D U E$ reuses the same cellular uplink channel that is assigned to the $l$-th cellular user equipment $\left(C U E_{l}\right)$. Moreover, the base station eNodeB has the control over the transmission power of the cellular user $C U E_{l}$ and of all $\mathrm{D} 2 \mathrm{D}$ user equipment to reduce the interference among them.

In Fig. 1, there are $M+3$ user equipment including one cellular user equipment and $M+2$ D2D user equipment. For simplicity, we name $D U E_{1}$ as node $0, R U E_{k}$ as node $k(k \in$ $\{1,2, \ldots, M\}), D U E_{2}$ as node $M+1$, and $C U E_{l}$ as node $M+2$. The notation $(i, j)(\forall i, j \in\{0,1, \ldots, M+1\}, i=j \pm 1)$ is used to represent the transmission link from node $i$ to node $j$.

\section{B. Channel model}

When node 0 , node $M+1$, and relay nodes in Fig. 1 reuse the same cellular uplink channel that is assigned to node $M+2$, they can be subject to the interfering signals from node $M+2$. A strong interference from node $M+2$ may result in frequent interruptions of the D2D links, which will have a serious impact on the successful reception rate of the desired signals at $\mathrm{D} 2 \mathrm{D}$ receiving nodes. Thus, the path loss and additive white Gaussian noise are introduced in our channel model. The large-scale fading is determined by the Euclidean distance $d_{i, j}$ between node $i$ and node $j$ together with path loss exponent $\alpha$, which is relevant to communication environments. The small-scale fading of link $(i, j)$ is characterized by a Rayleigh or Rician random variable $f_{i, j}$. Therefore, the instantaneous signal-to-interference-plus-noise ratio (SINR) at node $j$ with respect to node $i$ and node $M+2$ is given by

$$
\begin{aligned}
\gamma_{j} & =\frac{P_{T_{i}} \cdot d_{i, j}^{-\alpha} \cdot F_{i, j}}{P_{T_{M+2}} \cdot d_{M+2, j}^{-\alpha} \cdot F_{M+2, j}+\sigma^{2}} \\
& =\frac{F_{i, j}}{b \cdot c \cdot F_{M+2, j}+b \cdot \sigma^{2}},
\end{aligned}
$$

where $P_{T_{i}}$ is the transmit power of node $i, d_{i, j}^{-\alpha}$ is the path loss of link $(i, j)$ and $F_{i, j}=\left|f_{i, j}\right|^{2}$ is the channel gain of link $(i, j)$. Similarly, $P_{T_{M+2}}$ is the interference power of node $M+2$ with respect to node $j$. The notations $d_{M+2, j}^{-\alpha}$ and $F_{M+2, j}=\left|f_{M+2, j}\right|^{2}$ are the path loss and channel gain of link $(M+2, j)$, respectively. It is assumed that all nodes suffer the same additive white Gaussian noise $\sigma^{2}$. For convenience, we let $b=\frac{1}{P_{T_{i}} \cdot d_{i, j}^{-\alpha}}$ and $c=P_{T_{M+2}} \cdot d_{M+2, j}^{-\alpha}$ in (1).

Moreover, node 0 , node $M+1$, and relay nodes that form multiple D2D links are usually close next to each other, and this leads to the existence of dominant LoS signal components in the received desired signals. Also, these nodes are typically far away from node $M+2$, which results in no LoS signal components in the interference. Thus, in this paper, the Rician fading model is used for the channel gain of D2D links, and the Rayleigh fading model is used to deal with the interference links. We call such a setup Rician-Rayleigh channel fading model.

\section{Traffic model}

If the network traffic is not controlled properly, network congestions will occur and subsequently compromise the network throughput, resulting in an inferior network performance. Multi-hop D2D communication system should fully consider the traffic characteristics at the relay nodes. Therefore, the packet inter-arrival time distribution, packet length distribution, traffic burst, behavioral characteristics of short- or longterm traffic, and QoS requirements of various services should be taken into consideration, in order to more accurately analyze the entire multi-hop D2D communication system.

As illustrated in Fig. 1, each node has certain queue capacity and service capacity. We assume that node $j$ has a maximum queue capacity $Z_{j}$ (packets) to dynamically store arrival data packets, and each packet has the same length of $l$ (bits). Moreover, the transmission of data over the time is slot-by-slot based and each slot has a fixed duration $\Delta T$ (ms). In each time slot, the spectrum resource can be allocated to one or more D2D links, depending on the resource sharing and scheduling strategies. During each time slot, the average service rate at node $j, \mu_{j}$ (packets/ms), is upper bounded by the channel capacity of the corresponding D2D link. With network coding, a certain number of data packets have to be buffered for the decoding purpose, and such a number is smaller than queue capacity and is negligible in our analysis. In the following, we will discuss several typical network traffic models.

The Pareto traffic model is a self-similar model that mainly describes the traffic inter-arrival time with a heavy-tailed probability density function (pdf). The inter-arrival time of such traffic is independent and identically distributed. Specifically, 
the pdf of the inter-arrival time is the Pareto distribution given as follows [30]

$$
f(x)=\frac{S \cdot \beta^{S}}{x^{S+1}}, \quad(x \geq \beta)
$$

where $S$ is the shape parameter and $\beta$ is the minimum value of inter-arrival time. The mean and variance are respectively $\frac{S \cdot \beta}{S-1}$ and $\frac{S \cdot \beta^{2}}{(S-1)^{2}(S-2)}, 1<S<2$. A larger value of $S$ indicates a higher self-similarity level which can lead to a higher packet loss probability and a more serious deterioration of network performance.

The Gaussian traffic model is another option that works well with the traffic input process. Due to the huge capacity of network links, hundreds or even thousands of multimedia contents are likely to be served by a multiplexer. Therefore, even though the traffic from each individual application cannot be characterized as a Gaussian process, by appealing to the central limit theorem, the aggregate traffic to the receiver can be effectively modeled as a Gaussian process [31].

The fractional Brownian motion ( $\mathrm{fBm}$ ) traffic model is an important self-similar model that primarily describes the cumulative amount of traffic arrivals and models the variation of connectionless traffic with a self-similar Gaussian process. A standard $\mathrm{fBm}$ random process $Y(t)$ with Hurst parameter $H \in[0.5,1)$ is essentially a Gaussian process with the zero mean and the variance of $|t|^{2 H}$. As such, during each time slot, the cumulative amount of packets at node $j, A_{j}(\Delta T)$, which satisfies the self-similar fBm input traffic model, can be expressed as

$$
A_{j}(\Delta T)=\lambda_{j} \cdot \Delta T+\sqrt{\eta \cdot \lambda_{j}} \cdot Y(\Delta T),
$$

where $\eta$ is a variance coefficient, and $\lambda_{j}$ is the average packet arrival rate at node $j$ [32].

The Poisson traffic model, unlike other traffic models, is a conspicuous memoryless model (i.e., the future traffic behavior has nothing to do with the past traffic behavior). This model facilitates the traffic analysis process but cannot effectively reflect the burst nature of network traffic. During each time slot, the Poisson process of the cumulative amount of arrivals $A_{j}(\Delta T)$ at node $j$ with the parameter $\lambda_{j}$ is given by

$$
\operatorname{Pr}\left\{A_{j}(\Delta T)=n\right\}=e^{-\lambda_{j} \cdot \Delta T} \frac{\left(\lambda_{j} \cdot \Delta T\right)^{n}}{n !},
$$

where the expectation of $A_{j}(\Delta T)$ is equal to $\lambda_{j} \cdot \Delta T$ (i.e., $\left.E\left(A_{j}(\Delta T)\right)=\lambda_{j} \cdot \Delta T\right)$. Meanwhile, the packet inter-arrival time follows an exponential distribution with mean $1 / \lambda_{j}$. With a proper selection of parameters this model can be used to simulate most of the network traffic reasonably well for a small duration of time.

Many studies on network traffic analysis have shown that in real-world networks, network traffic when investigated over a long period of time typically possesses some features such as burst, self-similarity, and long-range dependence [33]. Consequently, when the network carries a large number of data packets from various sources, the self-similar traffic models such as Pareto and fBm traffic models would be appropriate; otherwise, the Poisson traffic model that does not show a clear traffic burst would be proper.

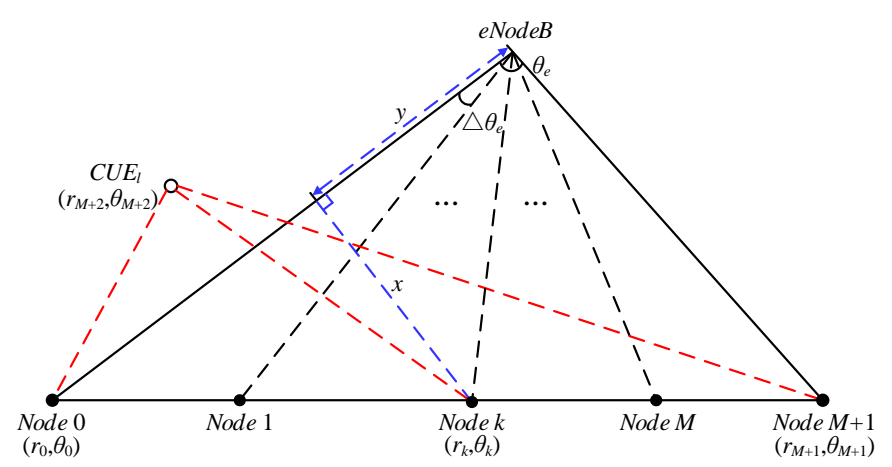

Fig. 2: The positioning of relay nodes $\left(\Delta \theta_{e}=\frac{\theta_{e}}{M+1}\right)$.

\section{The relay node selection and positioning}

Another critical issue in multi-hop D2D communications is the relay node selection strategy (see Fig. 1). We assume that all relay nodes are able to and willing to relay data packets in our communication scenario, and participate in the relay node selection by using their local information. As shown in Fig. 2, the polar coordinate system is used in selecting relay nodes. (The blue dashed lines in Fig. 2 are for the proof associated with this figure in Appendix.) The pole is the position of the base station eNodeB, and the polar axis is the horizontal ray going through eNodeB and pointing to east. In such a setting, the polar coordinate of any node $j$ would be $\left(r_{j}, \theta_{j}\right)$. It is assumed that each node is equipped with a GPS system and the eNodeB has the knowledge of the location information of node 0 and node $M+1$ initially. $M$ relay nodes are selected in the following way: each relay node must be on the straight line connecting node 0 and node $M+1$, and the polar coordinates $\left(r_{k}, \theta_{k}\right)$ of (the ideal) relay node $k(1 \leq k \leq M)$ are given by

$$
\begin{aligned}
& r_{k}=\frac{r_{0} \cdot r_{M+1} \cdot \sin \theta_{e}}{r_{0} \cdot \sin \frac{k \cdot \theta_{e}}{M+1}+r_{M+1} \cdot \sin \frac{(M+1-k) \theta_{e}}{M+1}} \\
& \theta_{k}=\frac{M+1-k}{M+1} \theta_{0}+\frac{k}{M+1} \theta_{M+1},
\end{aligned}
$$

where $\theta_{e}=\theta_{M+1}-\theta_{0}$. (A proof of (5) can be found in the Appendix of this paper.) Note that this relay node selection strategy can be characterized as the "shortest path" in the following sense. Assume that there are two candidate relay nodes $m$ and $m^{\prime}$ where $m$ is on the straight line connecting node 0 and node $M+1$, but $m^{\prime}$ is not. As such, by the laws of elementary geometry, the sum of the distance from node 0 to node $m^{\prime}$ and the distance from node $m^{\prime}$ to node $M+1$ will be longer than the sum of the distance from node 0 to node $m$ and the distance from node $m$ to node $M+1$. Therefore, the node on the straight line gives rise to the shortest possible distance. Since all relay nodes in our work are picked up on the straight line connecting node 0 and node $M+1$, we label such a strategy of choosing relay nodes as the "shortest path."

\section{D2D TRANSMISSION WITH NETWORK CODING AND ITS PACKET LOSS PROBABILITY}

\section{A. D2D transmission with network coding}

In this section, we investigate the situation that the data packets are transmitted via relay nodes with network coding 


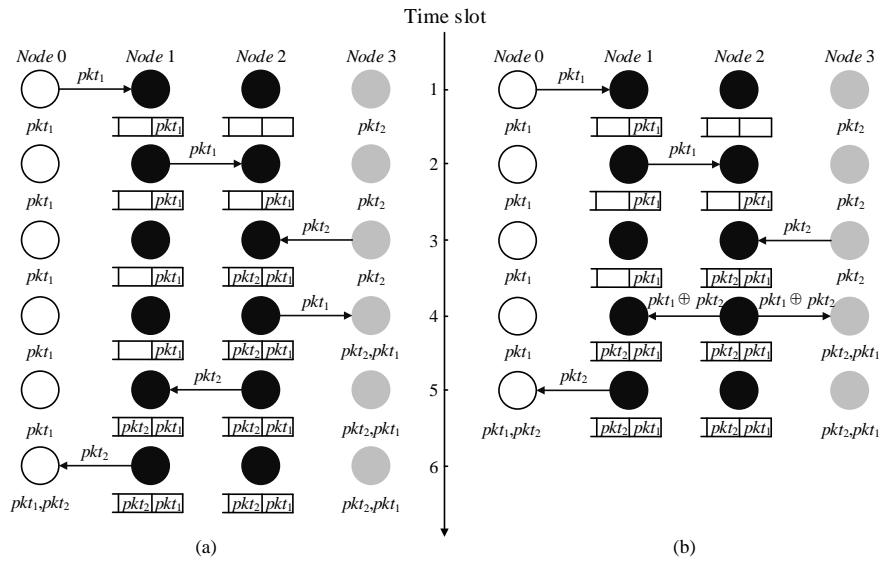

Fig. 3: An example of two-relay D2D packets exchange with or without XOR coding. (a) The packets exchange without XOR coding - 6 time slots required. (b) The packets exchange with XOR coding - 5 time slots required.

in multi-hop D2D links. When nodes 0 and $M+1$ need to exchange some multimedia contents with each other, the network coding mechanism [34], called XOR coding, can be used to improve the transmission efficiency. Note that the majority of the studies on network coding assisted transmissions only consider the special case of one relay node (or two-hop D2D links), whereas a generalized scenario of $M \geq 1$ relay nodes is tackled in our work. For the purpose of illustration, a special case of $M=2$ (i.e., two relay nodes) is depicted in Fig. 3.

In Fig. 3, node 0 and node 3 need to transmit source packets $\left(p k t_{1}\right.$ and $\left.p k t_{2}\right)$ to each other via relay nodes 1 and 2 , and node 2 (in Fig. 3(b)) is the relay where the XOR coding operation is designated to take place. Fig. 3(a) depicts the scenario where no XOR coding is used; clearly, 6 time slots are needed in this case to have $p k t_{1}$ and $p k t_{2}$ exchanged between node 0 and node 3. Fig. 3(b), on the other hand, illustrates the situation where XOR coding is used to assist the transmission. In Fig. 3(b), node 2, after receiving $p k t_{1}$ from node 0 (forwarded by node 1 ) and $p k t_{2}$ from node 3 , XORs the two and broadcasts the coded packet $p k t_{1} \oplus p k t_{2}$ to node 1 and node 3 . Node 3 obtains the needed $p k t_{1}$ by decoding the received coded packet; node 1 obtains the desired $p k t_{2}$ in a similar fashion and then forwards it to node 0 . As such, only 5 total time slots are needed to complete the exchange of the packets $p k t_{1}$ and $p k t_{2}$ between node 0 and node 3 . Based on this, it is easy to see that in the general case of $M$ relay nodes, the number of required time slots is $2 M+1$ with XOR coding, and $2(M+1)$ without XOR coding.

In order to reduce the complexity in using XOR coding, we mainly perform one-to-one encoding of source packets from node 0 and node $M+1$ through relay nodes. More specifically, suppose node 0 needs to transmit source packets $\left(p k t_{a 1}, p k t_{a 2}\right.$, $\left.p k t_{a 3}\right)$ to node $M+1$, for example, and node $M+1$ needs to send source packets $\left(p k t_{b 1}, p k t_{b 2}\right)$ to node 0 . Then, $p k t_{a 1}$ is only XORed with $p k t_{b 1}, p k t_{a 2}$ is only XORed with $p k t_{b 2}$, and so on. When node 0 needs to send more source packets than node $M+1$, the remaining source packets of node 0 (e.g. $\left.p k t_{a 3}\right)$ will no longer be XORed with source packets from node $M+1$. Instead, they will be transmitted directly in the multi-hop D2D links without being coded. Here, we assume that the D2D links have no packet loss. As such, when node $M+1$ and node 0 respectively receive $N_{1}$ and $N_{2}$ source packets, we can deduce that the total number of time slots with the presence of XOR coding is

$$
T_{s}^{c}=\left(N_{1}+N_{2}\right) \cdot M+\max \left\{N_{1}, N_{2}\right\} .
$$

Similarly, the total number of time slots without using XOR coding is

$$
T_{s}=\left(N_{1}+N_{2}\right) \cdot(M+1) .
$$

\section{B. Packet loss probability}

In Fig. 1 , when node 0 , node $M+1$, and the relay nodes which form the D2D links chain reuse the same cellular uplink channel, these nodes will be subject to the interference from node $M+2$. As mentioned before, this may lower the successful reception rate of desired signals at the receiving nodes since frequent D2D communication outages may occur due to the strong interference from cellular communications. On the other hand, note that network traffic also has an impact on the D2D data transmissions. Since the queue capacity and service capability of each relay node are limited, when the traffic amount exceeds a certain level, these relay nodes may start to malfunction causing network congestions and compromising the packet delivery. Therefore, in order to jointly deal with the impact from the cellular interference and network traffic conditions, we define the packet loss probability of D2D links as proposed in our previous work [22]:

$$
\operatorname{Pr}_{i, j}^{l}=\hat{\operatorname{Pr}}_{i, j} \cdot \operatorname{Pr}_{i, j}^{o}=\left(1-\overline{\operatorname{Pr}}_{i, j}\right) \cdot \operatorname{Pr}_{i, j}^{o},
$$

where $\operatorname{Pr}_{i, j}^{l}$ is the packet loss probability of link $(i, j) . \hat{\operatorname{Pr}}_{i, j}$ is the packet delivery probability defined to reflect traffic conditions over link $(i, j)$. $\overline{\mathrm{P}}_{i, j}$ is the packet failure probability, and $\operatorname{Pr}_{i, j}^{o}$ is the outage probability of link $(i, j)$.

Note that Eq. (8) is carefully constructed to model the packet loss probability of D2D links from two perspectives - network layer and physical layer. While $\operatorname{Pr}_{i, j}^{o}$ is a physical layer parameter that is determined by the channel condition and interference level, $\overline{\mathrm{P}}_{i, j}$ is a network layer parameter that indicates the network congestion caused by the traffic. It is reasonable to assume that $\operatorname{Pr}_{i, j}^{o}$ and $\bar{P}_{i, j}$ are independent of each other. In the following section, the closed-form expressions of $\operatorname{Pr}_{i, j}^{o}$ and $\operatorname{Pr}_{i, j}$ are respectively obtained using the Rician-Rayleigh channel fading model and various traffic models.

1) Link outage probability: The outage probability of transmission links needs to be studied on the basis of the relevant channel fading models. Most of the prior studies on D2D communications treat both the desired signals and the interference signals at the receiving nodes from the perspective of Rayleigh fading. This, however, is inaccurate since in closerange D2D communications a dominant part of the desired signals captured at the receiving nodes is actually the LoS signal. Thus, we use the Rician fading model in computing the the channel gain of D2D links, and the Rayleigh fading 
model in dealing with the interference signals received at the D2D nodes.

In (1), given that the envelope of the random variable $f_{i, j}$ has a Rician distribution, the pdf of the random variable $F_{i, j}$ has the exception value $\Omega$. Also, note that the envelope of the random variable $f_{M+2, j}$ has a Rayleigh distribution, which gives rise to its power being exponentially distributed. The pdf of the random variable $F_{M+2, j}$ has the exception value $\omega$. In the Rician-Rayleigh channel fading model, the link outage probability is defined as the probability that the instantaneous SINR at node $j$ in (1) is less than the SINR threshold $\gamma_{0}$, as follows $\operatorname{Pr}_{i, j}^{o}=\operatorname{Pr}\left(\gamma_{j}<\gamma_{0}\right)$. Therefore, the link outage probability under the Rician-Rayleigh fading model can be expressed as

$$
\begin{aligned}
\operatorname{Pr}_{i, j}^{o}= & \frac{(K+1) \gamma_{0}\left(1-\frac{\sigma^{2}}{c \cdot \omega+\sigma^{2}}\right)}{\theta+(K+1) \gamma_{0}\left(1-\frac{\sigma^{2}}{c \cdot \omega+\sigma^{2}}\right)} \\
& \times \exp \left[-\frac{K \cdot \theta}{\theta+(K+1) \gamma_{0}\left(1-\frac{\sigma^{2}}{c \cdot \omega+\sigma^{2}}\right)}+\frac{\sigma^{2}}{c \cdot \omega}\right],
\end{aligned}
$$

where $\theta=\frac{b \cdot c \cdot \Omega}{b \cdot c \cdot \omega+b \cdot \sigma^{2}}$ is a variable in $\mathrm{dB}, K$ is the Rician factor defined as the ratio of the power of the LoS signal components to that of the scattered signal components. Note that Eq. (9) can also express the link outage probability under the Rayleigh-Rayleigh fading model when $K=0$.

The proposed Rician-Rayleigh fading model might not be accurate when there are LoS signal components between the interfering node and the receiving node. However, a proper frequency channel assignment should avoid such a scenario. When the mean interfering power $c \cdot \omega$ is much larger than white Gaussian power $\sigma^{2}$, Eq. (9) can be rewritten as

$$
\operatorname{Pr}_{i, j}^{o} \approx \frac{(K+1) \gamma_{0}}{\theta+(K+1) \gamma_{0}} e^{-\frac{K \cdot \theta}{\theta+(K+1) \gamma_{0}}} .
$$

It is worth noting that this closed-form approximation is quite similar to the result of Yao and Sheikh [[35], Eq. (7)].

2) Packet failure probabilities: The packet failure probability is a network layer parameter that indicates the network congestion status caused by the traffic. When the queue of relay nodes reaches its capacity, the subsequent arriving packets will be dropped, which can lead to packet delivery failure. Let $Q_{j}(t)$ be the queue length (packets) of node $j$ at the beginning of the $t$-th time slot, which can be regarded as either a continuous-time or a discrete-time queue length. Here, $Q_{j}(t)$ is considered to be a continuous-time queue length because equivalent results can be obtained for the discrete-time queue length. At the beginning of the $(t+1)$-th time slot, the queue length $Q_{j}(t+1)$, the cumulative amount of arrival packets placed in the queue capacity, evolves as follows

$$
Q_{j}(t+1)=\max \left\{0, Q_{j}(t)-\mu_{j} \cdot \Delta T+A_{j}(\Delta T)\right\} .
$$

It is assumed that each node needs to send a signal for requesting data forwarding to the base station before each time slot, that the arrived source packets during each time slot can only be transmitted at the next time slot, and that the base station archives the sequence of the data forwarding requests. When the queue length of node $j$ exceeds its maximum capacity $Z_{j}, Q_{j}(\Delta T)=Q_{j}(t+1)-Q_{j}(t) \geq Z_{j}$, the packet delivery would fail. During the $t$-th time slot, the packet failure probability over link $(i, j), \overline{\operatorname{Pr}}_{i, j}$, is defined as

$$
\overline{\operatorname{Pr}}_{i, j}=\operatorname{Pr}\left\{A_{j}(\Delta T) \geq Z_{j}+\mu_{j} \cdot \Delta T\right\} .
$$

Next, we derive the closed-form expressions of $\overline{\mathrm{Pr}}_{i, j}$ with different traffic models.

The $\mathrm{fBm}$ traffic model formalizes the variation of connectionless traffic with a self-similar Gaussian process. By substituting (3) into (12), we see that the packet failure probability over link $(i, j)$ in $\mathrm{fBm}$ traffic model is

$$
\overline{\operatorname{P}} \mathrm{r}_{i, j}=1-\Phi\left(\frac{\left(\mu_{j}-\lambda_{j}\right) \cdot \Delta T+Z_{j}}{\sqrt{\eta \cdot \lambda_{j}} \cdot \Delta T^{H}}\right),
$$

where $\Phi(x)=\int_{-\infty}^{x} \varphi(y) d y$ is the cumulation distribution function of the standard Gaussian distribution, and $\varphi(x)=$ $\frac{1}{\sqrt{2 \pi}} e^{-\frac{x^{2}}{2}}$ is the probability density function. Note that $\Phi(x)$ can be similar to the formula $1-x^{-1} \varphi(x)$. We obtain the logarithmically asymptotical expression

$$
\overline{\operatorname{Pr}}_{i, j} \approx \frac{\sqrt{\eta \cdot \lambda_{j}} \cdot \Delta T^{H}}{\sqrt{2 \pi}\left[\left(\mu_{j}-\lambda_{j}\right) \cdot \Delta T+Z_{j}\right]} e^{-\frac{\left[\left(\mu_{j}-\lambda_{j}\right) \cdot \Delta T+Z_{j}\right]^{2}}{2 \eta \cdot \lambda_{j} \cdot \Delta T^{2 H}}} .
$$

Similarly, substituting (4) into (12) gives us the packet failure probability in the Poisson traffic model

$$
\overline{\operatorname{Pr}}_{i, j}=1-e^{-\lambda_{j} \cdot \Delta T} \cdot \sum_{n=0}^{Z_{j}+\mu_{j} \cdot \Delta T} \frac{\left(\lambda_{j} \cdot \Delta T\right)^{n}}{n !} .
$$

Since the Pareto traffic model is centered around the packet inter-arrival time, we cannot obtain an effective expression of cumulative amount of arrival packets. Fortunately, a Pareto/M/1 queueing model [36] through the use of the Pareto Laplace transform is studied. The packet failure probability under this queueing model is

$$
\begin{aligned}
\overline{\operatorname{Pr}}_{i, j}= & {\left[1-\frac{S(S-1)}{\rho} X^{\frac{S}{2}-1} e^{X / 2}\right.} \\
& \left.\times\left(\sqrt{X} \cdot W_{-\frac{S+1}{2},-\frac{S}{2}}(X)-W_{-\frac{S}{2},-\frac{1-S}{2}}(X)\right)\right] V^{Z_{j}}
\end{aligned}
$$

where $\rho=\frac{\lambda_{j}}{\mu_{j}}$ is the service utilization rate at node $j, W(\cdot)$ is the Whittaker's function, $X$ is equal to $\frac{(S-1) \cdot(1-V)}{\rho}$ and $V=$ $S \cdot X^{\frac{S-1}{2}} \cdot e^{X / 2} W_{-\frac{(S+1)}{2},-\frac{S}{2}}(X)$ is a geometric parameter.

\section{E2EPLP AND RELATED PERFORMANCE INDICATORS}

In the previous section, we analyzed the packet loss probability of D2D links from a local perspective. In this section, we analyze the performance of the entire system. The general closed-form expressions of the end-to-end packet loss probability with or without XOR coding on the basis of $\operatorname{Pr}_{i, j}^{l}$ in (8) are obtained. Then, the end-to-end completion time, the coding gain, and the average end-to-end transmission rate, are respectively investigated. 


\section{A. End-to-end packet loss probability}

In Fig. 3(b), the probability that node 2 can successfully encode packets sent from node 0 and node 3 , in time slot 3 , depends on the receiving condition of these packets at each of relay nodes. Only after successfully receiving the packets from node 1 (which has to successfully receive the packets from node 0 ) and node 3 , can node 2 perform the encoding process. In our work, without loss of generality, we assume that the packets encoding is done at the last relay node $M$. As such, the probability that relay node $M$ can successfully encode packets is

$$
\operatorname{Pr}_{M}^{c}=\operatorname{Pr}_{M+1, M}^{s} \cdot \prod_{i=1}^{M} \operatorname{Pr}_{i-1, i}^{s}, \quad(M \geq 1),
$$

where $\operatorname{Pr}_{i, j}^{s}=1-\operatorname{Pr}_{i, j}^{l}$ is the packet success probability of link $(i, j)$. Consequently, the probability that node $M+1$ fails to successfully receive the source packets from node 0 when node $M$ can encode the source packets is

$$
\operatorname{Pr}_{0, M+1}^{l, c}=1-\operatorname{Pr}_{M}^{c} \cdot \operatorname{Pr}_{M, M+1}^{s} .
$$

Analogously, the packet loss probability from node $M+1$ to node 0 is

$$
\operatorname{Pr}_{M+1,0}^{l, c}=1-\operatorname{Pr}_{M}^{c} \cdot \prod_{j=1}^{M} \operatorname{Pr}_{M+1-j, M-j}^{s} .
$$

When node $M+1$ correctly receives $N_{1}$ source packets, the total amount of source packets that node 0 needs to transmit is $\frac{N_{1}}{1-\mathrm{Pr}_{M+1,0}^{l, c}}$. Also, when node 0 correctly receives $N_{2}$ source packets, the total amount of source packets sent from node $M+1$ to node 0 is $\frac{N_{2}}{1-\operatorname{Pr}_{0}^{1, c}, c+1}$. Therefore, the system E2EPLP with the presence of XOR coding is

$$
\operatorname{Pr}_{E 2 E}^{l, c}=1-\frac{N_{1}+N_{2}}{\frac{N_{1}}{1-\operatorname{Pr}_{0, M+1}^{l, c}}+\frac{N_{2}}{1-\operatorname{Pr}_{M+1,0}^{l, c}}},
$$

which can be rewritten as follows by substituting (18) and (19) into (20)

$$
\begin{aligned}
& \operatorname{Pr}_{E 2 E}^{l, c}=1-\left(N_{1}+N_{2}\right) \prod_{i=0}^{M}\left(1-\operatorname{Pr}_{i, i+1}^{l}\right) \\
& \times \frac{\prod_{j=0}^{M}\left(1-\operatorname{Pr}_{M+1-j, M-j}^{l}\right)}{\prod_{j=1}^{M}\left(1-\operatorname{Pr}_{M+1-j, M-j}^{l}\right) N_{1}+\left(1-\operatorname{Pr}_{M, M+1}^{l}\right) N_{2}} .
\end{aligned}
$$

Then, the system E2EPLP in the absence of XOR coding can be given by

$$
\operatorname{Pr}_{E 2 E}^{l}=1-\frac{\left(N_{1}+N_{2}\right)\left(1-\operatorname{Pr}_{0, M+1}^{l}\right)\left(1-\operatorname{Pr}_{M+1,0}^{l}\right)}{\left(1-\operatorname{Pr}_{M+1,0}^{l}\right) N_{1}+\left(1-\operatorname{Pr}_{0, M+1}^{l}\right) N_{2}},
$$

where $\operatorname{Pr}_{0, M+1}^{l}=1-\prod_{i=0}^{M}\left(1-\operatorname{Pr}_{i, i+1}^{l}\right)$ is the packet loss probability from node 0 to node $M+1, \operatorname{Pr}_{M+1,0}^{l}=1-$
$\prod_{j=0}^{M}\left(1-\operatorname{Pr}_{M+1-j, M-j}^{l}\right)$ is the packet loss probability from node $M+1$ to node 0 . In other words,

$$
\begin{aligned}
\operatorname{Pr}_{E 2 E}^{l}= & 1-\left(N_{1}+N_{2}\right) \prod_{i=0}^{M}\left(1-\operatorname{Pr}_{i, i+1}^{l}\right) \\
\times & \frac{\prod_{j=0}^{M}\left(1-\operatorname{Pr}_{M+1-j, M-j}^{l}\right)}{\prod_{j=0}^{M}\left(1-\operatorname{Pr}_{M+1-j, M-j}^{l}\right) N_{1}+\prod_{i=0}^{M}\left(1-\operatorname{Pr}_{i, i+1}^{l}\right) N_{2}}
\end{aligned}
$$

By examining Eqs. (21) and (23), we can see that the mechanism of XOR coding and the number of relays have impacts on the system E2EPLP. Since the encoding/decoding operations of XOR coding occur at some relay nodes, the probability of these operations being successfully/unsuccessfully performed has to be an extra part of the computation of system E2EPLP when the coding scheme is present. Moreover, considering that the packet loss process on each single-hop D2D link can be regarded as an independent Bernoulli event, an increased number of D2D links will inevitably increase the system E2EPLP. Similarly, a larger number of relay nodes would yield a higher system E2EPLP.

\section{B. Other related performance indicators}

In Section III, we saw that the total required number of time slots for the receiving node to correctly receive source packets is $T_{s}^{c}$. Note that it was computed under the assumption that there is no packet loss during the transmission, which means that the number of received source packets at the destination node is equal to the number of source packets sent at the source node. Here, we revisit the transmission time of source packets taking the packet loss probability into consideration. Using the aforementioned E2EPLP, the end-to-end completion time with XOR coding, $T_{E 2 E}^{c}$, which indicates the total transmission time required for correctly decoding packets at the destination node, can be calculated as

$$
T_{E 2 E}^{c}=\frac{T_{s}^{c} \cdot \Delta T}{1-\operatorname{Pr}_{E 2 E}^{l, c}} .
$$

In a similar fashion, the end-to-end completion time without XOR coding can be formulated as $T_{E 2 E}=\frac{T_{s} \cdot \Delta T}{1-\operatorname{Pr}_{E 2 E}^{l}}$. Note that the computation time required for encoding and decoding the source packets during the transmission is ignored in the above calculations.

The notion of coding gain [34] is defined the as the ratio of the total required packet transmission time without the presence of coding to that with the presence of coding (for the same set of packets). Thus, for the XOR coding, the coding gain would be

$$
G=\frac{T_{E 2 E}}{T_{E 2 E}^{c}},
$$

which reflects the benefits of XOR coding. Specifically, a larger the value of coding gain indicates a stronger benefit.

In addition, a new performance indicator, average end-toend transmission rate, is introduced in [37]. In our XOR 


\section{TABLE II: PARAMETER SETTINGS}

\begin{tabular}{lc}
\hline Parameter Description & Value \\
\hline The SINR threshold $\gamma_{0}(\mathrm{~dB})$ & -10 \\
Reused uplink bandwidth $B(\mathrm{MHz})$ & 2 \\
Rician factor $K(\mathrm{~dB})$ & 7 \\
Path loss exponent $\alpha$ & 4 \\
The time slot duration $\Delta T(\mathrm{~ms})$ & 10 \\
The average service rate $\mu_{j}(\mathrm{packets} / \mathrm{ms})$ & 0.4 \\
The maximum queue capacity $Z_{j}$ (packets) & 40 \\
Packet size $l(\mathrm{~KB})$ & 1 \\
The coefficient of variance $\eta$ & 1 \\
Hurst parameter $H$ & 0.7 \\
Pareto shape parameter $S$ & 1.5 \\
The number of relay nodes $M$ & $1,3,5$ \\
The number of source packets $N_{1}, N_{2}$ & 100,50 \\
\hline
\end{tabular}

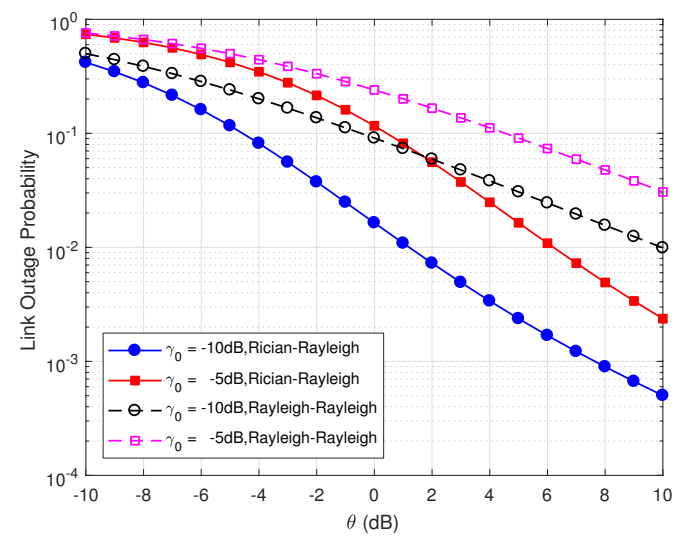

Fig. 4: D2D link outage probability with different values of $\theta$ and channel fading models.

coding, when node 0 correctly receives $N_{2}$ source packets, and node $M+1$ correctly receives $N_{1}$ source packets, the corresponding average end-to-end transmission rate can be defined as

$$
R_{E 2 E}^{c}=\frac{N_{1}+N_{2}}{T_{E 2 E}^{c}},
$$

which captures the number of packets transmitted per time unit over the multi-hop D2D links.

\section{Simulation RESUlT}

In this section, we conduct extensive simulations to analyze the performance of the proposed multi-hop D2D communication model with respect to the variation of several parameters. All parameters used in the simulations are shown in Table II.

Fig. 4 shows the D2D link outage probability in different channel fading models with respect to the value of $\theta$. Without loss of generality, we randomly select two SINR thresholds $\gamma_{0}=-10 \mathrm{~dB}$ and $\gamma_{0}=-5 \mathrm{~dB}$ in the simulations. In closerange D2D communications, a dominant part of the desired signals captured at the D2D receiving nodes is actually the LoS signal. Therefore, the Rician-Rayleigh channel fading model should be used. Here, the Rician factor $K$ is set to be $7 \mathrm{~dB}$. Note that a larger Rician factor indicates stronger LoS signal components, and that $K=0$ in fact entails that the link outage probability under the Rayleigh-Rayleigh channel fading model is to be computed. Clearly, we can see from Fig. 4

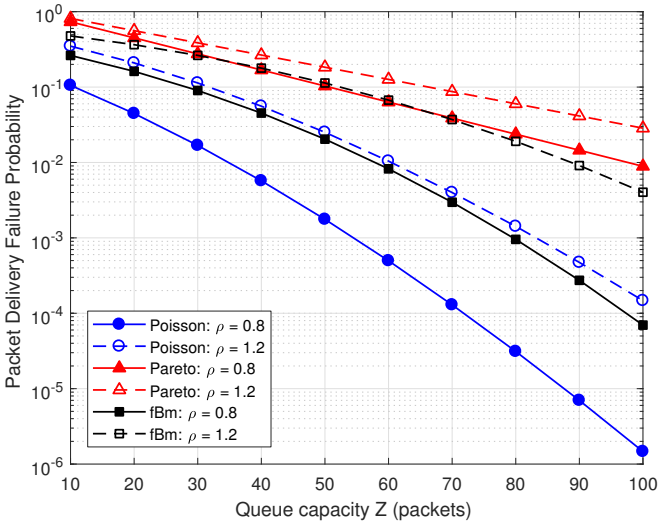

(a)

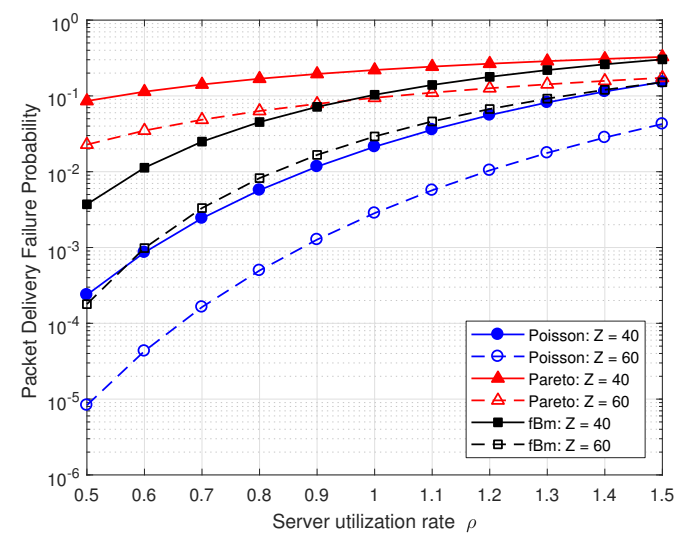

(b)

Fig. 5: Packet failure probability with different traffic models.

that for any fixed SINR threshold the link outage probability decreases as the $\theta$ increases, that a larger threshold leads to a higher link outage probability, and that the Rician-Rayleigh channnel fading model which takes the LoS signal components into account yields a lower link outage probability than the Rayleigh-Rayleigh channel fading model. In order to keep the link outage probability low and facilitate the subsequence analyses, $\gamma_{0}$ is set to be $-10 \mathrm{~dB}$ for the rest of the simulations.

Fig. 5 illustrates the packet failure probability in Pareto, $\mathrm{fBm}$, and Poisson traffic models, and with respect to the queue capacity $Z$ and node service utilization rate $\rho$. While the queue capacity reflects the relay nodes' ability to dynamically store arrival packets, the service utilization rate is defined to be the ratio of the packet arrival rate $\lambda_{j}$ to the service rate $\mu_{j}$. When the queue capacity is full or $\lambda_{j}>\mu_{j}$, the arriving packets are more likely to be discarded. As it can be seen in Fig. 5, a low queue capacity is more likely to result in a packet delivery failure, and $\rho>1\left(\lambda_{j}>\mu_{j}\right)$ tends to cause more packet delivery failures than $\rho<1$. To facilitate the subsequence analyses, $Z$ is randomly chosen to be 40 for the rest of the simulations. Moreover, it is worth noting that the packet failure probabilities of the Pareto and fBm traffic models, which take the burst and the self-similarity of network traffic as part of their consideration, are higher than that of the Poisson traffic model.

In a similar fashion, Fig. 6 depicts the packet loss probabil- 


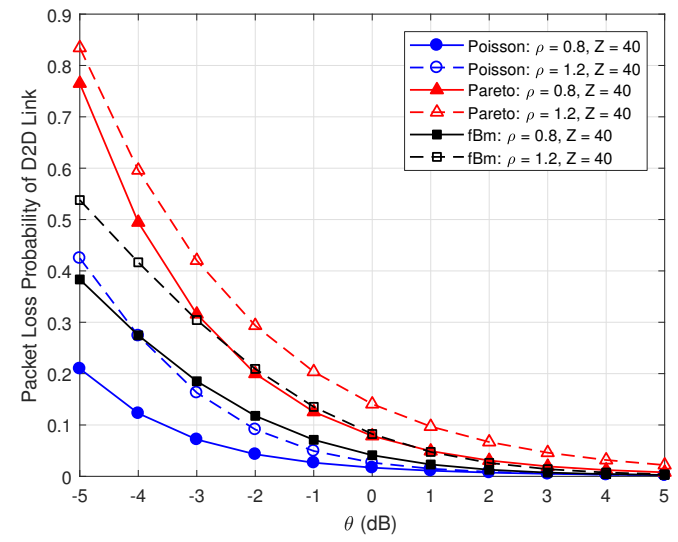

Fig. 6: Packet loss probability of D2D links in different traffic models.

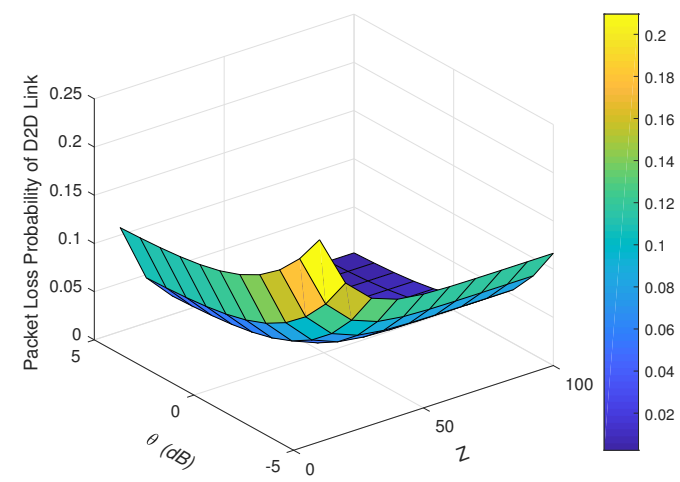

(a)

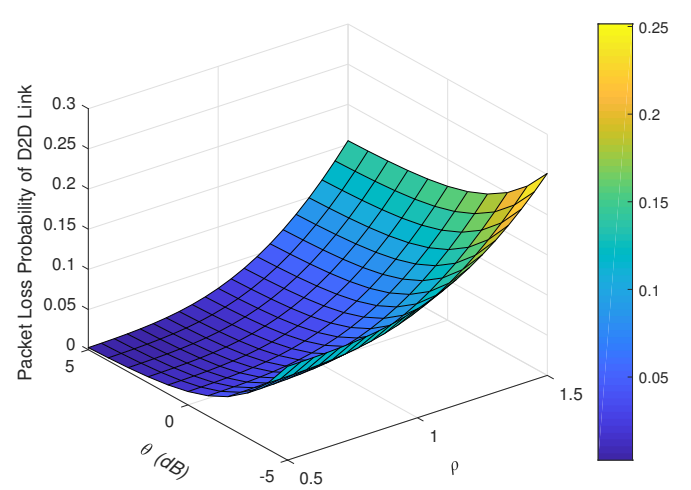

(b)

Fig. 7: Packet loss probability of D2D links in Poisson traffic model.

ity of D2D links, which is constructed from the outage probability and packet failure probability, in the aforementioned traffic models. Again, it can be observed that the packet loss probability in Poisson traffic model is smaller than that in the other two traffic models. Note that in the multi-hop D2D communication scenario described in Fig. 1, node 0 and node $M+1$ typically transmit a small amount of source packets to each other, which indicates that there would typically be less network traffic burst between node 0 and node $M+1$. Thus, we focus on the study of the packet loss probability using the Poisson traffic model for the rest of the simulations.

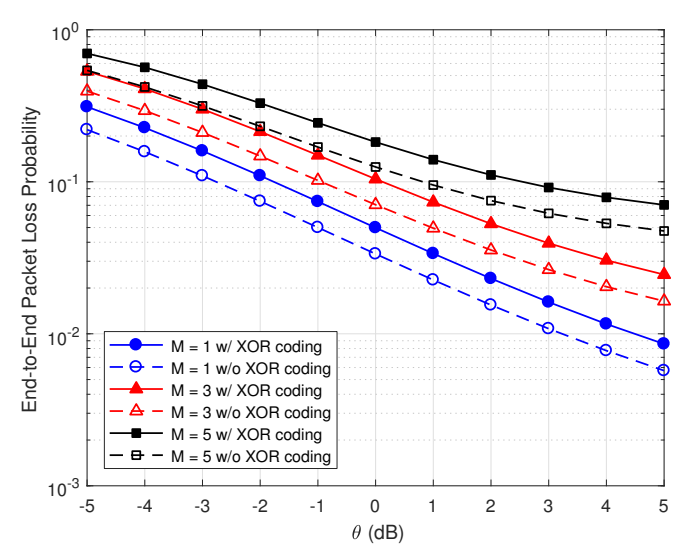

(a)

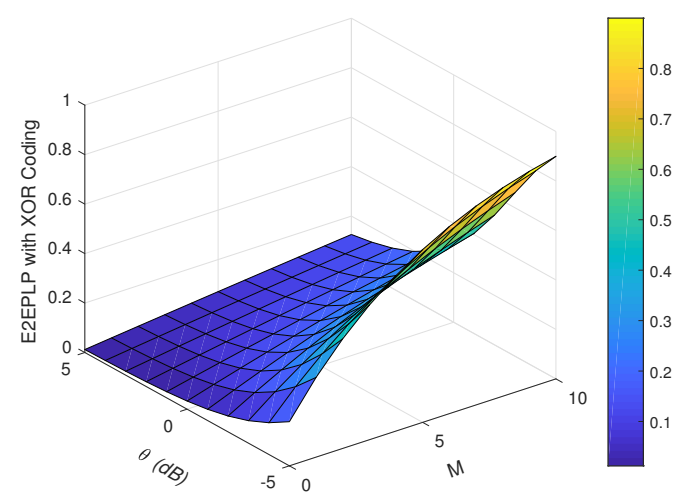

(b)

Fig. 8: System E2EPLP with or without XOR coding in Poisson traffic model.

In Fig. 7, which shows the packet loss probability in Poisson traffic model indexed by $\theta$ and queue capacity $Z$, and by $\theta$ and service utilization rate $\rho$. We see that packet loss probabilities are higher when $\theta \in[-5,0]$ and $Z \in[0,50]$ (Fig. 7(a)), and when $\theta \in[-5,0]$ and $\rho \in[1,1.5]$ (Fig. 7(b)). For the sake of convenience of comparison, we assume that the packet loss probabilities of all D2D links between node 0 and node $M+1$ in Fig. 1 are the same.

Fig. 8 depicts the system E2EPLP with respect to the presence/absence of XOR coding and the number of relay nodes $M$. Interestingly, we can see, in Fig. 8(a), that the mechanism of XOR coding actually raises the system E2EPLP. This should not be a surprise as XOR coding in fact increases the amount of information in the packets that are being transmitted, which, in turn, would result in an increase in the packet loss probability. Also, since the encoding/decoding operations of XOR coding occur at some relay nodes, the probability of these operations being successfully/unsuccessfully performed has to be (an extra) part of the computation of the system E2EPLP when the coding scheme is present. As for the number of relay nodes $M$, considering that the packet loss process on each single-hop D2D link can be regarded as an independent Bernoulli event, then, an increased number of D2D links (or of relays) will inevitably increase the system E2EPLP. On the other hand, an increased communication distance between the two D2D end users will certainly lead 


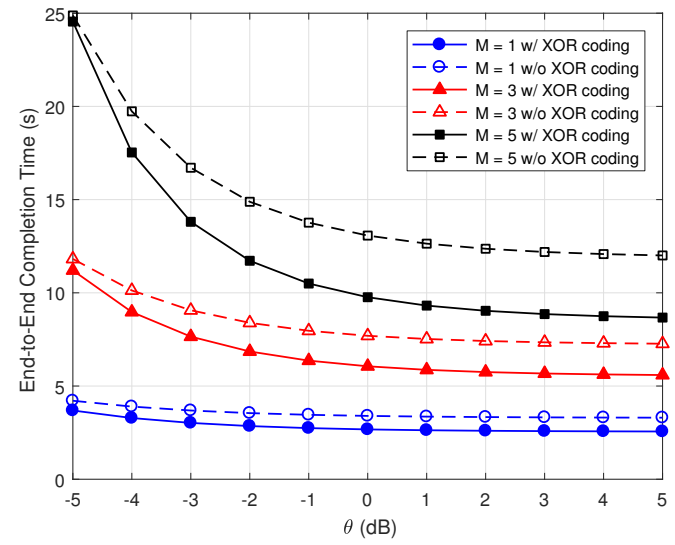

Fig. 9: System end-to-end completion time with and without XOR coding in Poisson traffic model.

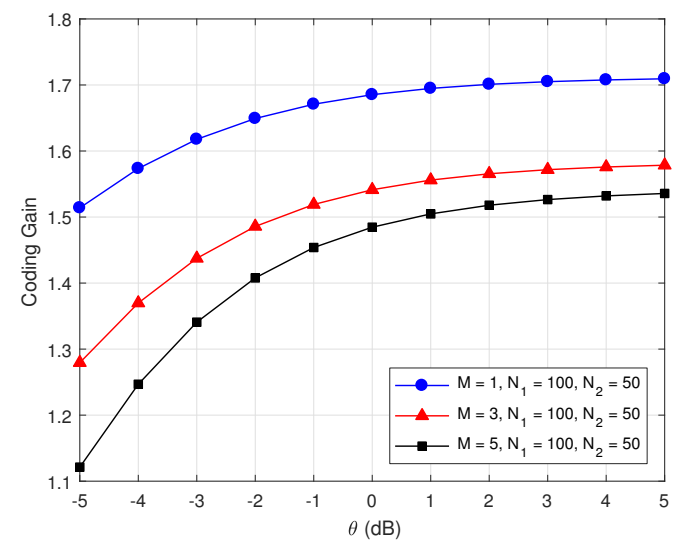

Fig. 10: Coding gain in Poisson traffic model.

to a worse signal fading and will consequently affect the successful reception of signals. These explain why a larger number of relay nodes yields a higher system E2EPLP in Fig. 8.

We now investigate the the following performance indicators of the system: end-to-end completion time, coding gain, and average end-to-end transmission rate. In doing so, we set the numbers of required source packets $\left(N_{1}\right.$ and $\left.N_{2}\right)$ for nodes $M+1$ and 0 to be able to correctly receive, to be 100 and 50 , respectively. Note that this setting is reasonable in the sense that although a huge amount of packets are being transmitted in some networks on a daily basis, the amount of packets transmitted in D2D communications is typically small.

Fig. 9 depicts the system end-to-end completion time in Poisson traffic model with the presence/absence of XOR coding and with different number of relay nodes. Clearly, we see that the end-to-end completion time when the XOR coding scheme is present is lower than that when the XOR coding scheme is absent. This is due to the fact that the mechanism of XOR coding can reduce the number of needed time slots for packet transmissions, and a reduced number of time slots of course yields a less amount of packet transmission time. Also, Fig. 9 indicates that a larger number of relay nodes $M$ leads to a higher end-to-end completion time. The reason for this is that a lager number of relay nodes will increase the

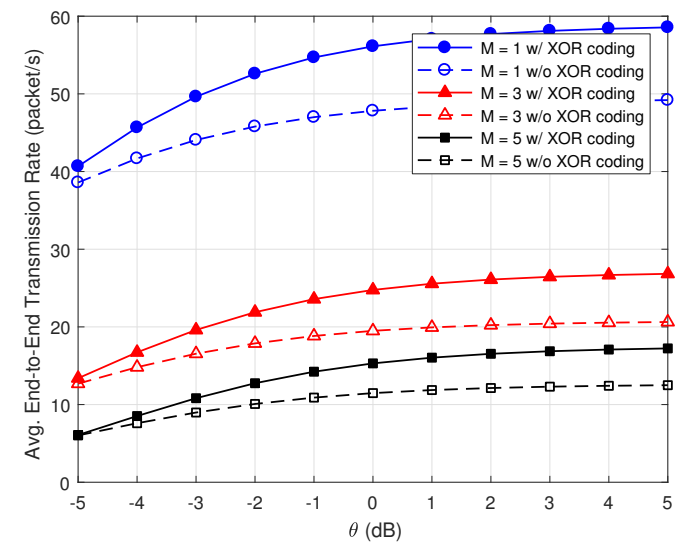

Fig. 11: System average end-to-end transmission rate with and without XOR coding in Poisson traffic model.

end-to-end packet loss probability, which, in turn, will lower the probability of packet being successfully decoded at the receiving end, and eventually increase the system end-to-end completion time. In a similar fashion, Fig. 10 shows the system coding gain in Poisson traffic model with different number of relay nodes. Since the end-to-end completion time with the presence of XOR coding is always less than that without XOR coding, the system coding gain by its definition will be always greater than 1.

Fig. 11 exhibits the system average end-to-end transmission rates with the presence/absence of XOR coding and with the number of relay nodes, when $N_{1}$ and $N_{2}$ are fixed. The result that the system average end-to-end transmission rate with the presence of XOR coding is greater than that with the absence of XOR coding, as shown in Fig. 11, can be attributed to the fact that the mechanism of XOR coding reduces the end-toend completion time of the system. Another result from the Fig. 11 is that an increase in the number of relay nodes will result in a decrease in the average end-to-end transmission rate. This is actually what is expected, as a larger number of relays will yield a longer end-to-end completion time.

Fig. 12 (a) and (b) depict the comparison of our work (XOR coding) with RLNC and batch coding [37] in terms of end-toend completion time and average end-to-end transmission rate, respectively. Since the framework of the D2D communications in [37] is two-hop (i.e., one relay node), we set $M=1$ in our work for a fair comparison. As shown in Fig. 12(a), the endto-end completion time of our work is the highest one. This is not surprising as encoding of packets is conducted in a batch mode in both RLNC and batch coding schemes (i.e., either $N_{1}$ source packets are encoded at node 0 or multiple packets buffered at the relay nodes are encoded and then forwarded), but only in a one-to-one fashion in our work. This fact allows both RLNC and batch coding use much less time slots than the XOR coding, resulting in a much less end-to-end completion time than our work, as shwon in Fig. 12(a). Fig. 12(b) can be understood in a similar way since the end-to-end transmission rate is inversely proportional to the end-to-end completion time.

Note that although the performance of XOR coding is worse 


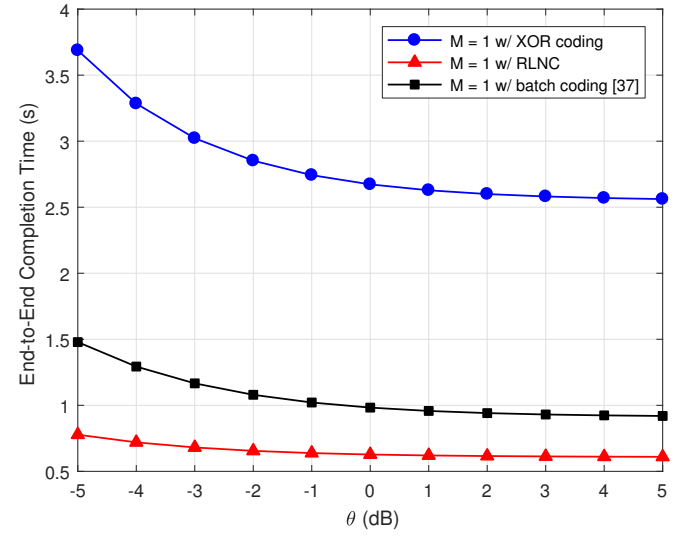

(a)

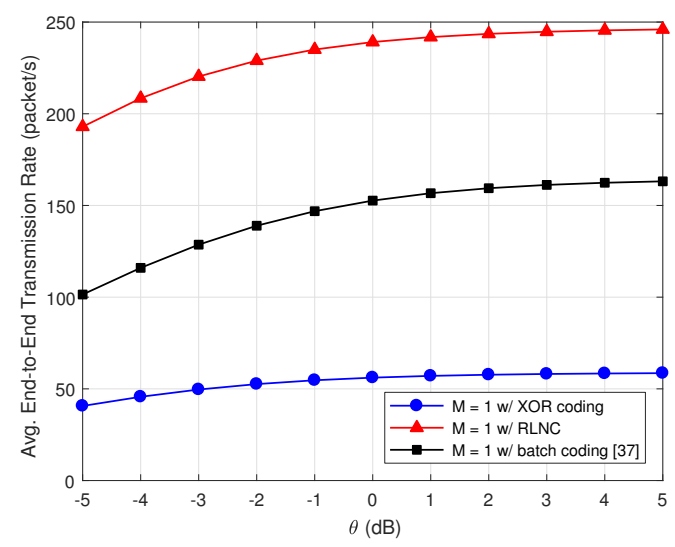

(b)

Fig. 12: Comparison of the effect of various network coding in Poisson traffic model.

than that of the other two coding approaches in terms of end-to-end completion time and end-to-end transmission rate, the implementation of XOR coding is much simpler than that of the other two approaches. This simplicity is actually preferred when energy efficiency is much of a concern in D2D communications in which all mobile devices are subject to energy constraints.

\section{CONCLUSION}

We have proposed a general network coding-assisted multihop D2D communication system underlaying the cellular network, together with its associated relay node selection strategy, in which the base station determines the position of all relay nodes by using the location information of the D2D pair and in conformity with the criterion of the "shortest path". In the proposed system, both Rician-Rayleigh channel fading model and Rayleigh-Rayleigh channel fading model are investigated. It turns out that the Rician-Rayleigh channel fading model, which takes the LoS signal components into consideration, outperforms the Rayleigh-Rayleigh channel fading model in terms of D2D link outage probability. Also, the packet delivery status in different traffic models were thoroughly analyzed. We have found that Pareto and $\mathrm{fBm}$ traffic models, which include traffic properties such as burst and self-similarity as an essential part in their formations, yield a higher packet failure probability than that of the Poisson traffic model. In addition, the system E2EPLP with the presence or absence of network coding were computed using the packet loss probability of D2D links, and we have noticed that the network coding scheme and the number of relays both can impact the performance of the system. In particular, three critical system performance indicators, namely, E2EPLP, end-to-end completion time, and average end-to-end transmission rate are all subject to the presence/absence of the network coding scheme and the change in the number of relays.

\section{APPENDIX A}

PROOF OF FORMULA (5)

In Fig. 2, $x$ and $y$ are the two legs of the right triangle formed by eNodeB, node $k$, and the line segment that goes through node $k$ and is perpendicular to the line connecting eNodeB and node 0.

Note that

$$
\tan \angle N \text { ode } 0=\frac{x}{r_{0}-y} \text { and } \tan \left(k \Delta \theta_{e}\right)=\frac{x}{y},
$$

from which can obtain

$$
y=\frac{r_{0} \cdot \tan \angle N o d e 0}{\tan \left(k \Delta \theta_{e}\right)+\tan \angle N o d e 0} .
$$

Thus,

$r_{k}=\frac{y}{\cos \left(k \Delta \theta_{e}\right)}=\frac{r_{0} \cdot \tan \angle N o d e 0}{\sin \left(k \Delta \theta_{e}\right)+\tan \angle N o d e 0 \cdot \cos \left(k \Delta \theta_{e}\right)}$.

Note also that

$$
\tan \angle N o d e 0=\frac{r_{M+1} \cdot \sin \theta_{e}}{r_{0}-r_{M+1} \cdot \cos \theta_{e}},
$$

so the polar coordinates $\left(r_{k}, \theta_{k}\right)$ of relay node $k$ are

$$
\begin{aligned}
r_{k} & =\frac{r_{0} \cdot r_{M+1} \cdot \sin \theta_{e}}{r_{0} \cdot \sin \frac{k \cdot \theta_{e}}{M+1}+r_{M+1} \cdot \sin \frac{(M+1-k) \theta_{e}}{M+1}} \\
\theta_{k} & =\theta_{0}+\frac{k}{M+1} \theta_{e} .
\end{aligned}
$$

\section{REFERENCES}

[1] P. Li, S. Guo, and I. Stojmenovic, "A truthful double auction for device-to-device communications in cellular networks," IEEE Journal on Selected Areas in Communications, vol. 34, no. 1, pp. 71-81, Jan. 2016.

[2] B. Klaiqi, X. Chu, and J. Zhang, "Energy- and spectral-efficient adaptive forwarding strategy for multi-hop device-to-device communications overlaying cellular networks," IEEE Transactions on Wireless Coтmиnications, vol. 17, no. 9, pp. 5684-5699, Sept. 2018.

[3] B. Ying and A. Nayak, "A power-efficient and social-aware relay selection method for multi-hop D2D communications," IEEE Cоттиnications Letters, vol. 22, no. 7, pp. 1450-1453, July 2018.

[4] Z. Zhang, P. Zhang, D. Liu, and S. Sun, "SRSM-based adaptive relay selection for D2D communications," IEEE Internet of Things Journal, vol. 5, no. 4, pp. 2323-2332, Aug. 2018.

[5] I. Krikidis, T. Charalambous, and J. S. Thompson, "Buffer-aided relay selection for cooperative diversity systems without delay constraints," IEEE Transactions on Wireless Communications, vol. 11, no. 5, pp. 1957-1967, May 2012.

[6] A. Zanella, A. Bazzi, and B. M. Masini, "Relay selection analysis for an opportunistic two-hop multi-user system in a Poisson field of nodes," IEEE Transactions on Wireless Communications, vol. 16, no. 2, pp. 1281-1293, Feb. 2017. 
[7] Y. Yan, B. Zhang, and C. Li, "Network coding aided collaborative real-time scalable video transmission in D2D communications," IEEE Transactions on Vehicular Technology, vol. 67, no. 7, pp. 6203-6217, July 2018

[8] Y. Keshtkarjahromi, H. Seferoglu, R. Ansari, and A. Khokhar, "Deviceto-device networking meets cellular via network coding," IEEE/ACM Transactions on Networking, vol. 26, no. 1, pp. 370-383, Feb. 2018.

[9] J. Huang, S. Huang, and C.-C. Xing, "When D2D meets network coding: A tutorial case study," in Proceedings of International Conference on Research in Adaptive and Convergent Systems (RACS), Krakow, Poland, Sep. 2017.

[10] J. Huang, H. Gharavi, H. Yan, and C. C. Xing, "Network coding in relay-based device-to-device communications," IEEE Network, vol. 31 no. 4, pp. 102-107, July 2017.

[11] L. Wei, R. Q. Hu, Y. Qian, and G. Wu, "Energy efficiency and spectrum efficiency of multihop device-to-device communications underlaying cellular networks," IEEE Transactions on Vehicular Technology, vol. 65, no. 1, pp. 367-380, Jan. 2016.

[12] C. Gao, Y. Li, Y. Zhao, and S. Chen, "A two-level game theory approach for joint relay selection and resource allocation in network coding assisted D2D communications," IEEE Transactions on Mobile Computing, vol. 16, no. 10, pp. 2697-2711, Oct. 2017.

[13] Y. Zhao, Y. Li, X. Chen, and N. Ge, "Joint optimization of resource allocation and relay selection for network coding aided device-to-device communications," IEEE Communications Letters, vol. 19, no. 5, pp. 807-810, May 2015.

[14] M. S. Karim, A. Douik, S. Sorour, and P. Sadeghi, "Rate-aware network codes for completion time reduction in device-to-device communications," in Proceedings of IEEE International Conference on Communications (ICC), Kuala Lumpur, Malaysia, May 2016.

[15] A. Douik, S. Sorour, T. Y. Al-Naffouri, and M. Alouini, "Instantly decodable network coding: From centralized to device-to-device communications," IEEE Communications Surveys \& Tutorials, vol. 19, no. 2 , pp. 1201-1224, Secondquarter 2017.

[16] Y. Wu, W. Liu, S. Wang, W. Guo, and X. Chu, "Network coding in device-to-device (D2D) communications underlaying cellular networks," in Proceedings of IEEE International Conference on Communications (ICC), London, UK, June 2015.

[17] E. Datsika, A. Antonopoulos, N. Zorba, and C. Verikoukis, "Crossnetwork performance analysis of network coding aided cooperative outband D2D communications," IEEE Transactions on Wireless Communications, vol. 16, no. 5, pp. 3176-3188, May 2017.

[18] J. Huang, S. Huang, C. C. Xing, and Y. Qian, "Game-theoretic power control mechanisms for device-to-device communications underlaying cellular system," IEEE Transactions on Vehicular Technology, vol. 67, no. 6, pp. 4890-4900, June 2018 .

[19] X. Xu, Y. L. Guan, and Y. Zeng, "Batched network coding with adaptive recoding for multi-hop erasure channels with memory," IEEE Transactions on Communications, vol. 66, no. 3, pp. 1042-1052, March 2018.

[20] P. Li, S. Guo, S. Yu, and A. V. Vasilakos, "Reliable multicast with pipelined network coding using opportunistic feeding and routing," IEEE Transactions on Parallel and Distributed Systems, vol. 25, no. 12, pp. 3264-3273, Dec. 2014.

[21] S. Dang, G. Chen, and J. P. Coon, "Multicarrier relay selection for full-duplex relay-assisted OFDM D2D systems," IEEE Transactions on Vehicular Technology, vol. 67, no. 8, pp. 7204-7218, Aug. 2018.

[22] J. Huang and H. Gharavi, "Performance analysis of relay-based twoway D2D communications with network coding," IEEE Transactions on Vehicular Technology, vol. 67, no. 7, pp. 6642-6646, July 2018.

[23] N. B. Mehta, V. Sharma, and G. Bansal, "Performance analysis of a cooperative system with rateless codes and buffered relays," IEEE Transactions on Wireless Communications, vol. 10, no. 4, pp. 10691081, April 2011.

[24] D. D. Penda, R. S. Risuleo, P. E. Valenzuela, and M. Johansson, "Optimal power control for D2D communications under Rician fading: A risk theoretical approach," in Proceedings of IEEE Global Communications Conference (GLOBECOM), Singapore, Dec. 2017.

[25] M. Lin, J. Ouyang, and W. P. Zhu, "Joint beamforming and power control for device-to-device communications underlaying cellular networks," IEEE Journal on Selected Areas in Communications, vol. 34 no. 1, pp. 138-150, Jan. 2016.

[26] M. Peng, Y. Li, T. Q. S. Quek, and C. Wang, "Device-to-device underlaid cellular networks under Rician fading channels," IEEE Transactions on Wireless Communications, vol. 13, no. 8, pp. 4247-4259, Aug. 2014.
[27] J. Kim, S. Kim, J. Bang, and D. Hong, "Adaptive mode selection in D2D communications considering the bursty traffic model," IEEE Communications Letters, vol. 20, no. 4, pp. 712-715, April 2016.

[28] S. Huang, B. Liang, and J. Li, "Distributed interference and delay aware design for D2D communication in large wireless networks with adaptive interference estimation," IEEE Transactions on Wireless Communications, vol. 16, no. 6, pp. 3924-3939, June 2017.

[29] L. Lei, X. Shen, M. Dohler, C. Lin, and Z. Zhong, "Queuing models with applications to mode selection in device-to-device communications underlaying cellular networks," IEEE Transactions on Wireless Communications, vol. 13, no. 12, pp. 6697-6715, Dec. 2014.

[30] X. Bai and A. Shami, "Modeling self-similar traffic for network simulation," CoRR, vol. abs/1308.3842, 2013, http://arxiv.org/abs/1308.3842. [Online]. Available: http://arxiv.org/abs/1308.3842

[31] J. Choe and N. B. Shroff, "A central-limit-theorem-based approach for analyzing queue behavior in high-speed networks," IEEE/ACM Transactions on Networking, vol. 6, no. 5, pp. 659-671, Oct. 1998.

[32] I. Norros, "On the use of fractional Brownian motion in the theory of connectionless networks," IEEE Journal on Selected Areas in Communications, vol. 13, no. 6, pp. 953-962, Aug. 1995.

[33] K. Wang, X. Li, H. Ji, and X. Du, "Modeling and optimizing the LTE discontinuous reception mechanism under self-similar traffic," IEEE Transactions on Vehicular Technology, vol. 65, no. 7, pp. 5595-5610, July 2016.

[34] S. Katti, H. Rahul, W. Hu, D. Katabi, M. Medard, and J. Crowcroft, "Xors in the air: Practical wireless network coding," IEEE/ACM Transactions on Networking, vol. 16, no. 3, pp. 497-510, June 2008.

[35] Y. D. Yao and A. U. H. Sheikh, "Outage probability analysis for microcell mobile radio systems with cochannel interferers in Rician/Rayleigh fading environment," Electronics Letters, vol. 26, no. 13, pp. 864-866, June 1990.

[36] R. M. Rodrfguez-Dagnino, "Some remarks regarding asymptotic packet loss in the Pareto/M/1/K queueing system," IEEE Communications Letters, vol. 9, no. 10, pp. 927-929, Oct. 2005.

[37] Y. Li, S. Zhang, J. Wang, X. Ji, H. Wu, and Z. Bao, "A lowcomplexity coded transmission scheme over finite-buffer relay links," IEEE Transactions on Communications, vol. 66, no. 7, pp. 2873-2887, July 2018 .

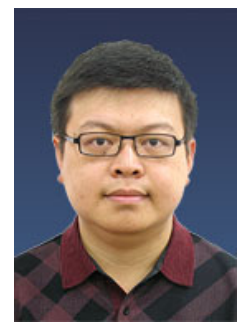

Jun Huang (M'12-SM'16) received the Ph.D degree (with honor) from the Institute of Network Technology, Beijing University of Posts and Telecommunications, China, in 2012. He is a full professor of computer science with the Chongqing University of Posts and Telecommunications. Dr. Huang was a visiting scholar in the Global Information and Telecommunication Institute, Waseda University, a research fellow in the Electrical and Computer Engineering Department, South Dakota School of Mines and Technology, a visiting scholar in the Computer Science Department, the University of Texas at Dallas, and a guest professor at the National Institute of Standards and Technology. $\mathrm{He}$ received the outstanding service award from ACM RACS 2017, the runnerup of best paper award from ACM SAC 2014, and the best paper award from AsiaFI 2011. He has authored 100+ publications including papers in prestigious journal and conferences. He is an Associate Editor of IEEE Access and the KSII Transactions on Internet and Information Systems. He guest-edited several special issues on IEEE journals such as the IEEE Communications Magazine, the IEEE Internet of Things Journal, the IEEE Access, etc. He also chaired and co-chaired multiple conferences in the communications and networking areas and organized multiple workshops at major IEEE and ACM events. His current research interests include network optimization and control, D2D communications, and the Internet of Things. $\mathrm{He}$ is a senior member of the IEEE. 


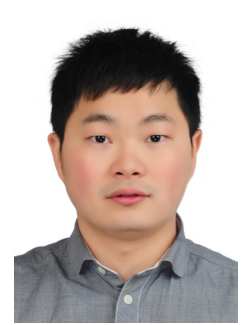

Yong Liao is current a master student in Chongqing University of Posts and Telecommunications, his research interest lies in network coding and D2D communications.

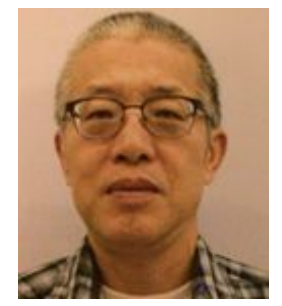

Cong-Cong Xing is a Professor of Computer Science/Mathematics at Nicholls State University, Thibodaux, Louisiana, USA. He received his Ph.D. in Computer Science and Engineering from $\mathrm{Tu}-$ lane University, New Orleans, USA, joining the Nicholls State University faculty in 2001. His research interests include theoretical foundations of programming languages, category theory, and mobile/wireless computing and analysis. He is active in research in these areas.

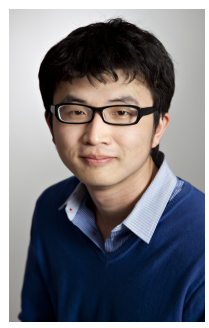

Zheng Changg (S'10-M'13-SM'17) received the B.Eng. degree from Jilin University, Changchun, China in 2007, M.Sc. (Tech.) degree from Helsinki University of Technology (Now Aalto University), Espoo, Finland in 2009 and Ph.D degree from the University of Jyväskylä, Jyväskylä, Finland in 2013. Since 2008, he has held various research positions at Helsinki University of Technology, University of Jyväskylä and Magister Solutions Ltd in Finland. During June to August in 2013, he was a visiting researcher at Tsinghua University, China and during April to May in 2015, he was a visiting researcher at University of Houston, TX. He has been awarded by the Ulla Tuominen Foundation, the Nokia Foundation and the Riitta and Jorma J. Takanen Foundation for his research excellence.

He serves as editor of IEEE Access, Springer Wireless Networks and IEEE MMTC Communications Frontier, and guest editor of IEEE Wireless Communications, IEEE Communications Magazine, IEEE Internet of Things Journal, EURASIP Journal on Wireless Communications and Networking, Physical Communications and Wireless Communications and Mobile Computing. $\mathrm{He}$ also served as TPC member for many IEEE major conferences, such as INFOCOM, ICC and Globecom. He has received Best Paper awards from IEEE Technical Committee on Green Communications \& Computing (TCGCC) and 23rd Asia-Pacific Conference on Communications (APCC) in 2017. $\mathrm{He}$ was also named the exemplary reviewer of IEEE Wireless Communications Letter in 2017. Currently he is working as Assistant Professor at University of Jyväskylä and his research interests include IoT, cloud/edge computing, security and privacy, vehicular networks, and green communications. 